\title{
Plastic Deformation of Oriented Lamellae. II. Hot Rolling of $\beta$-Phase Isotactic Polypropylene
}

\author{
Tsutomu ASANO, Yasuna FuJIWARA, and Toshiho Yoshida \\ Department of Physics, Faculty of Science, Shizuoka University, \\ Shizuoka 422, Japan.
}

(Received August 25, 1978)

\begin{abstract}
Isotactic polypropylene was crystallized under temperature gradient and an oriented $\beta$-phase sample was obtained. The $\beta$-phase film was rolled in three orthogonal directions at $68,96,122$, and $142^{\circ} \mathrm{C}$. The deformation behavior was investigated by wide angle and small angle $\mathrm{X}$ ray diffraction methods. Anisotropy in deformation was observed in each case, including the rotation of lamellae, interlamellar slip, longitudinal and transversal chain slip. At high temperatures, the deformation proceeded mainly by interlamellar slip whereas chain slip could hardly be observed. During the hot roll deformation, the original $\beta$-phase crystal was destroyed and then recrystallized into the $c$-axis-oriented $\alpha$-phase crystal. The amount of the $\beta \rightarrow \alpha$ phase transition increased with increasing rolling temperature and draw-ratio. The spacing of the newly appeared $\alpha$-phase lamellae changed, depending only on the rolling temperature. The final $c$-axis-oriented texture was composed of $\alpha$-phase crystals. From these results, it is considered that the deformation proceeds, not by incorporation of the original lamellar blocks, but through the melting or unfolding of the original $\beta$ phase lamellae and subsequent recrystallization into the $c$-axis-oriented $\alpha$-phase lamellae.

KEY WORDS Hot Rolling / Polypropylene / X-Ray / Crystallization / Temperature Gradient / Oriented Lamellae / $\beta \rightarrow \alpha$ Phase Transition / Interlamellar Slip / Chain Slip / Melt and Recrystallization /
\end{abstract}

In a previous paper, the structural change during cold rolling of oriented $\beta$-phase isotactic polypropylene was reported. ${ }^{1}$ The oriented $\beta$-phase sample was crystallized from the melt under temperature gradient ("Seto method"). ${ }^{2}$ The study of these samples helped greatly to interpret the anisotropic character of lamellar deformation because of their crystalline orientation. Also, when the $\beta$-phase crystals (hexagonal lattice) are destroyed or melted, they recrystallized into the more thermally stable $\alpha$-phase crystal (monoclinic lattice). Thus, it is easy to estimate by X-ray diffraction methods the degree of destruction of the $\beta$-phase crystals during deformation. The previous paper showed that deformation takes place by rotation of lamellae, interlamellar slip, longitudinal and transversal chain slip, and also that the $\beta$-phase crystals are destroyed during deformation and that the final $c$-axis-oriented texture is composed of $\alpha$-phase crystals.

In general, the deformation mechanism of polymers changes with temperature because of the change in the plasticity of the sample. Several papers have reported the effect of temperature on plastic deformation, showing that temperature influences the attainable draw ratio, the transparency of the drawn material, the long period and intensity observed in the small angle X-ray diffraction pattern, etc. $^{3-5}$ In the case of isotactic polypropylene $\beta$-phase crystals, it is also expected that the deformation mechanism changes with the temperature. Though the $c$-axis-oriented $\beta$-phase crystal could not be observed in the cold rolling experiment, it may be that the $\beta$-phase crystal can be incorporated into the final $c$-axis-oriented texture at higher temperatures. Thus, the deformation of isotactic polypropylene $\beta$ phase crystals by hot rolling was studied at temperatures of $68,96,122$, and $142^{\circ} \mathrm{C}$, by wide angle X-ray scattering (WAXS) and small angle $X$-ray scattering (SAXS).

\section{STARTING MATERIAL}

An oriented $\beta$-phase sample film was made from a commercial isotactic polypropylene sheet given from 


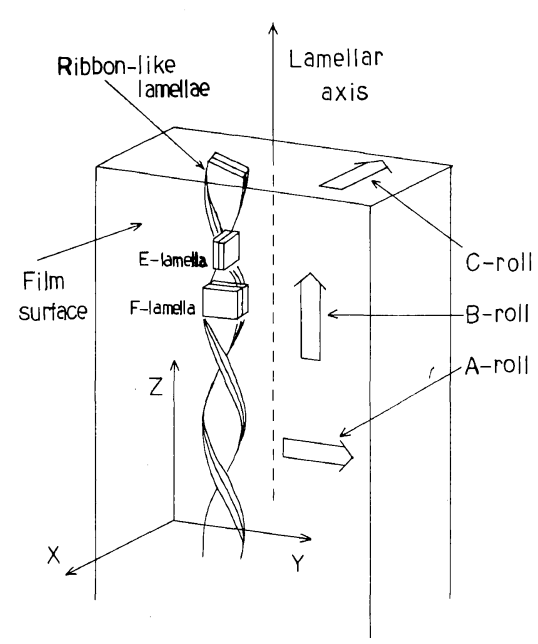

Figure 1. Ribbon-like lamellae and three rolling directions, and definition of Cartesian coordinates relative to a sample plate.

Chisso Co. Ltd. The sample sheet was moved slowly (about $0.5 \mathrm{~mm} / \mathrm{h}$ ) under temperature gradient. In a previous paper, ${ }^{1}$ the oriented growth method was reported and also, the orientation of the isotactic polypropylene $\beta$-phase crystal, schematically shown in Figure 1. In the sample film, ribbon-like lamellae grew along the crystallizing direction (the lamellar axis). The crystalline $a$-axis was parallel to this direction, and the $c$-axis was always perpendicular to it and rotated helically around it. Accordingly, this sample was almost the same as the one cut out from a $\beta$-phase spherulite along its radius.

The average measured density of the $\beta$-phase sample was 0.900 . The fraction of crystals in the sample was obtained from the measured density by the expression,

$$
x_{\mathrm{c}}=d_{\mathrm{c}}\left(d-d_{\mathrm{am}}\right) / d\left(d_{\mathrm{c}}-d_{\mathrm{am}}\right)
$$

where, $d$ is the measured density, $d_{\mathrm{am}}$ is the amorphous density $\left(0.858 \mathrm{~g} / \mathrm{cm}^{3}\right)$, and $d_{\mathrm{c}}$ is the crystal density $\left(0.921 \mathrm{~g} / \mathrm{cm}^{3}\right){ }^{6}$ The average calculated crystallinity of the sample was $68 \%$, which is nearly the same as that observed by Samuels, et al., from the $\beta$ phase spherulites $(72 \%){ }^{6}$

\section{EXPERIMENTAL}

Cartesian coordinates are designated in the sample film, as shown in Figure 1. The $X$-axis is perpendicular to the film surface, the $Y$-axis parallel to the film surface and perpendicular to the lamellar axis and the $Z$-axis is parallel to the lamellar axis. These axes will also be used without change following the deformation.

Samples were rolled in the following three ways. A-roll: Roll plane parallel to $Y Z$ plane and roll direction parallel to $Y$-axis.

B-roll: Roll plane parallel to $Y Z$ plane and roll direction parallel to $Z$-axis.

C-roll: Roll plane parallel to $X Y^{\prime}$ plane and roll direction parallel to $X$-axis.

The original film thickness was about $250 \mu \mathrm{m}$ in the case of the A- and B-rolls, but in the case of the Croll, strips of about $250 \mu \mathrm{m}$ in height were cut out parallel to the $X Y$ plane from the original film of 800 $\mu \mathrm{m}$ thick, and these strips were subjected to rolling.

The rolling deformation was carried out at temperatures of $68,96,122$, and $142^{\circ} \mathrm{C}$. The results obtained by the cold rolling were compared. The rolling temperature was measured directly by the thermocouple inserted into the polymer film placed between temperature controlled rollers. The diameter of the rollers was $7.5 \mathrm{~cm}$. The sample film was deformed by repeated rolling, gradually narrowing the gap until desired draw ratio was attained. The rolling speed was about $1 \mathrm{~mm} / \mathrm{s}$. Draw ratio $\lambda$ was measured by the change of length between marks made beforehand in the original sample. X-ray patterns with the incident $\mathrm{X}$-ray beam parallel to $X$-, $Y$-, and $Z$-axes were denoted as $X$-, $Y$-, and $Z$ patterns, respectively. All $X$-ray photographs were taken at room temperature in the unclamped state after rolling. The pinhole diameters of the WAXS and SAXS cameras were 0.2 and $0.3 \mathrm{~mm}$, respectively. The camera distance of the former was $30 \mathrm{~mm}$ and that of the latter, $360 \mathrm{~mm}$. Rigaku RU3 apparatus was used as the X-ray source.

\section{RESULTS}

\section{A-Roll Deformation}

WAXS $Z$-patterns of the samples A-rolled at 68, 96, 122, and $142^{\circ} \mathrm{C}$ are shown in Figure 2. The draw ratio of each of these samples is about 3 . Prior to the roll deformation, the $\beta$-phase (300) reflection of the $Z$-pattern is a uniform ring, representing the uniform distribution of the $c$-axis around the $Z$-axis. In the 
Plastic Deformation of Oriented Lamellae. II.

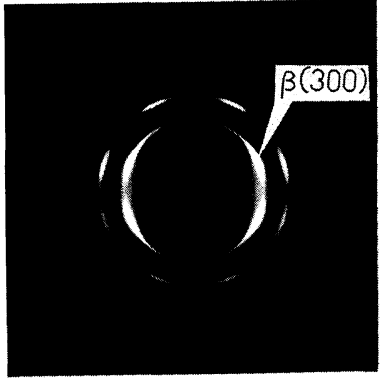

a

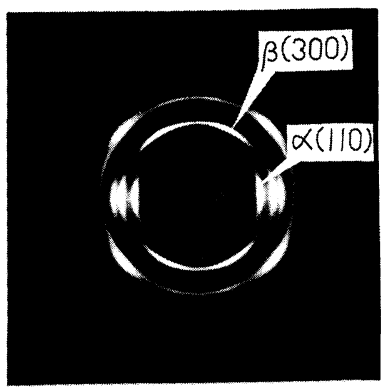

d

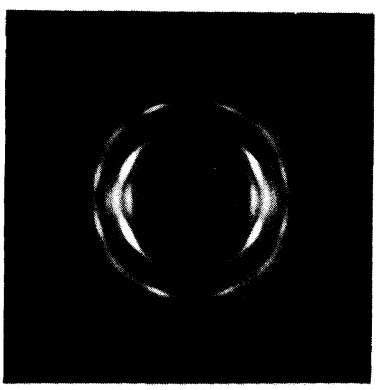

b

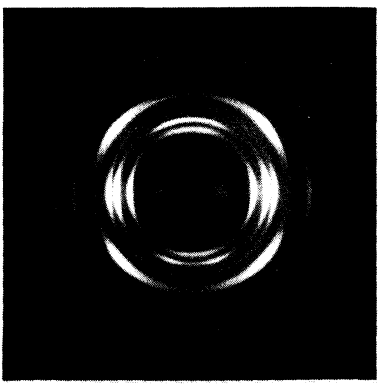

e

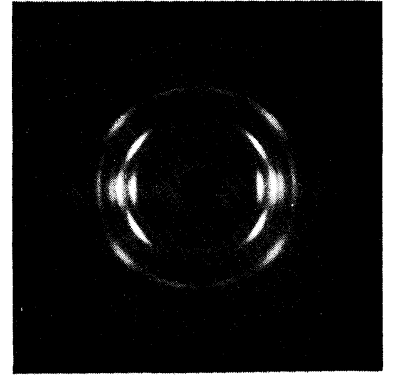

C

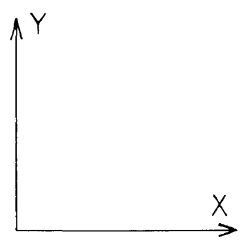

Figure 2. WAXS $Z$-patterns of the samples A-rolled al various temperatures: a, at $20^{\circ} \mathrm{C}, \lambda=3.2 ; \mathrm{b}$, at $68^{\circ} \mathrm{C}$, $\lambda=2.8 ; \mathrm{c}$, at $96^{\circ} \mathrm{C}, \lambda=3.0 ; \mathrm{d}$, at $122^{\circ} \mathrm{C}, \lambda=3.0$; e, at $142^{\circ} \mathrm{C}, \lambda=3.0$. The roll direction ( $Y$-axis) is vertical.

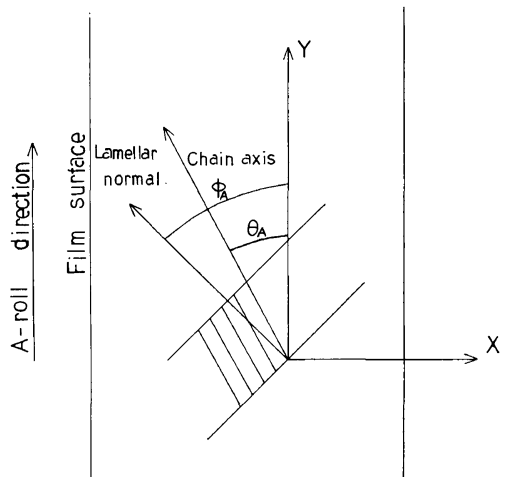

Figure 3. Definition of $\theta_{\mathrm{A}}$ and $\phi_{\mathrm{A}}$ in the A-roll deformation.

case of the rolling deformation of $\lambda=3.2$ at room temperature, the $\beta$-phase (300) reflection shows four arcs near the equator (Figure 2a). This pattern was analysed in the previous paper, and it was shown that the $c$-axis is inclined by $25^{\circ}$ from the $Y$-axis as a result of interlamellar slip and chain slip. In the A-roll deformation at room temperature, both lamellar and chain orientations change mostly in the $X Y$ plane, and so consequently, mainly the SAXS and WAXS
$Z$-patterns have been investigated. In the case of hot roll deformation, the SAXS and WAXS Z-patterns are also very important for examining lamellar and chain orientation.

In Figure 2, it is seen that the intensity of the $\beta$ phase (300) reflection decreases with increasing temperature. This fact indicates that, at nearly the same stage of deformation $(\lambda \sim 3)$, the $\beta$-phase crystallites are destroyed more easily at a higher temperature. The angle between the $c$-axis and the roll direction ( $Y$-axis) can be measured from the orientation of the (300) reflection. As shown in Figure 3 , this angle is denoted as $\theta_{\mathrm{A}}$. The results are shown in Table I, where $\theta_{\mathrm{A}}$ increases with rolling temperature. The $c$-axis of the $\beta$-phase crystal approaches a position parallel to the rolling direction at lower temperatures, but even at room temperature, it makes $25^{\circ}$ with the $Y$-axis. With increasing rolling temperature, the $c$-axis approaches a position perpendicular to the rolling direction. The $c$-axisorientation of the $\beta$-phase crystal can not be observed within temperature range of this experiment.

There is a diffuse peak on the equator of Figure 2a, which originated from imperfect $\alpha$-phase micro- 
Table I. Values of $\theta_{\mathrm{A}}$ and $\phi_{\mathrm{A}}$ as a function of rolling temperature

\begin{tabular}{ccccc}
\hline $\begin{array}{c}\text { Roll temp, } \\
{ }^{\circ} \mathrm{C}\end{array}$ & $\lambda$ & $\begin{array}{c}\theta_{\mathrm{A}}, \\
\text { degree }\end{array}$ & $\begin{array}{c}\phi_{\mathrm{A}}, \\
\text { degree }\end{array}$ & $\begin{array}{c}\phi_{\mathrm{A}}-\theta_{\mathrm{A}}, \\
\text { degree }\end{array}$ \\
\hline 20 & 3.2 & 25 & 75 & 50 \\
68 & 2.8 & 27 & 70 & 43 \\
96 & 3.0 & 40 & 73 & 33 \\
122 & 3.0 & 60 & 78 & 18 \\
142 & 3.0 & 70 & - & - \\
\hline
\end{tabular}

crystallites formed during the roll deformation at room temperature. At elevated temperatures, several crystalline reflections analyzed as $h k 0$ reflections of the $\alpha$-phase crystal can be observed on the equator. These reflections appear in the course of rolling deformation, and their intensity increases with the rolling temperature. As a result of examining the $X$-, $Y$-, and $Z$-patterns for each sample, the $\alpha$-phase crystal is seen to have a so-called $c$-axis-orientation with the $c$-axis parallel to the rolling direction ( $Y$ axis). At $142^{\circ} \mathrm{C}$, however, the $\alpha$-phase (110) arc also appears near the meridian, which seems to arise from the difference in crystalline orientation. There are several papers on the meridional (110) reflection which is considered to originate from the $a^{*}$-axisoriented $\alpha$-phase crystallites. ${ }^{7,8}$

In the SAXS $Z$-patterns observed in hot roll deformation of $\lambda \sim 3$, the diagonal reflections from the $\beta$-phase lamellae grew weaker with increasing roll temperature, but they could be observed up to $122^{\circ} \mathrm{C}$. The angle $\phi_{\mathrm{A}}$, which is the angle between the $\beta$-phase lamellar normal and the roll direction as shown in Figure 3, was observed from diagonal spots of the SAXS $Z$-pattern. As shown in Table I, $\phi_{\mathrm{A}}$ is about $70-80^{\circ}$ for all observable temperatures. Accordingly, the angle $\phi_{\mathbf{A}}-\theta_{\mathbf{A}}$, representing the inclination of $c$-axis in the $\beta$-phase lamella, decreases with rolling temperature. At $142^{\circ} \mathrm{C}$, the angle $\phi_{\mathrm{A}}$ could not be measured since no diagonal spots were seen in the SAXS pattern; but supposing it to be the same as that observed at other temperatures, $\phi_{\mathrm{A}}-\theta_{\mathrm{A}}$ will become nearly zero.

The above results show that, at lower roll temperatures, the $c$-axis of the $\beta$-phase crystal is tilted within the lamellae as shown in Figure 4a. At higher temperatures, the deformation proceeds mainly by interlamellar slip, and tilt deformation hardly occurs, as shown in Figure 4b. It is speculated that in the case of deformation at higher temperatures, the non-crystalline part surrounding the crystalline lamellae becomes soft, and the lamellae are easily rotated by interlamellar slip. On the other hand, the lamellae themselves are apt to be destroyed because of the enhanced partial melting. Accordingly, the lamellae are destroyed before chain slip deformation takes place.

During hot roll deformation, new reflections appear on the meridian of the SAXS Z-pattern, whose intensity increases with increasing roll temperature in the same manner as the WAXS $\alpha$-phase reflections. Long spacing estimated from SAXS meridional reflections increases with increasing rolling temperature as shown in Table II. The long spacings, measured from the samples rolled at room temperature and subsequently annealed at 100 and $120^{\circ} \mathrm{C}$, show good agreement with those obtained from hot rolled samples at 96 and $122^{\circ} \mathrm{C}$, respectively. From these facts, it is considered that the $\alpha$-phase lamellae are formed during hot roll deformation and that the lamellar thickness changes with the rolling temperature.

To clarify the deformation process during hot rollings, SAXS and WAXS Z-patterns during the Aroll deformation at $142^{\circ} \mathrm{C}$ are shown in Figure 5 at three stages of $\lambda$. At $\lambda=1.3$, the SAXS diffraction becomes weak on the meridional direction and maximum intensity is observed on the diagonal position (about $\pm 45^{\circ}$ from the $Y$-axis). A corresponding WAXS $Z$-pattern at $\lambda=1.3$ shows that the $\alpha$-phase (300) reflection becomes weak on the equatorial direction and that there are several weak diffraction arcs from $\alpha$-phase crystallites.

At $\lambda=2.0$, new peaks appear on the SAXS me-

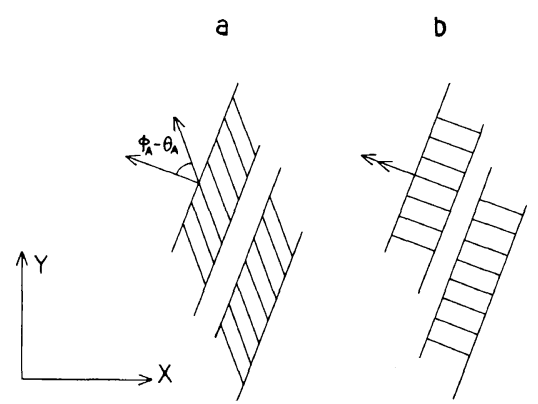

Figure 4. Schematic diagram showing both lamellar and chain orientation. a: at lower temperature; the $\beta$ phase $c$-axis is tilted within the lamella. b: at higher temperature; the $\beta$-phase $c$-axis is parallel to the lamellar normal. 
Plastic Deformation of Oriented Lamellae. II.

Table II. Long spacing of $\alpha$-phase lamellae

\begin{tabular}{cc}
$\begin{array}{c}\text { Roll temp, } \\
{ }^{\circ} \mathrm{C}\end{array}$ & $\begin{array}{c}\text { Long spacing, } \\
\AA\end{array}$ \\
\hline $20^{\mathrm{a}}$ & $130 \sim 140$ \\
$20^{\mathrm{b}}$ & 170 \\
68 & $120 \sim 140$ \\
96 & 150 \\
122 & 180 \\
142 & 250
\end{tabular}

a The sample was rolled at $20^{\circ} \mathrm{C}$ and thereafter annealed under tension for $2 \mathrm{~h}$ at $100^{\circ} \mathrm{C}$.

b The sample was rolled at $20^{\circ} \mathrm{C}$ and thereafter annealed under tension for $2 \mathrm{~h}$ at $120^{\circ} \mathrm{C}$.

ridian whereas the diagonal spots from $\beta$-phase lamellae grow weaker than those observed at $\lambda=1.3$. The diagonal spots seem to lie on the ellipsoid. At $\lambda=3.0$, no SAXS diagonal reflections are observed, whereas meridional reflections grow stronger. In the WAXS pattern at $\lambda=2.0$, several $\alpha$-phase $h k 0$ diffraction arcs are seen near the equator indicating that the $c$-axis of the $\alpha$-phase crystallites is nearly parallel to the roll direction ( $Y$-axis). On the other hand, diffractions from $\beta$-phase (300) planes make arcs spreading between $\pm 45^{\circ}$ from the meridian. At $\lambda=3.0$, these arcs change to weak peaks near the meridian showing that the angle between the $\beta$-phase $c$-axis and $X$-axis is $70^{\circ}$ as analysed in Table $\mathrm{I}$. The intensity of the $\beta$-phase (300) peaks decreases with increasing $\lambda$. On the other hand, the strong $\alpha$-phase (110) peaks at $\lambda=3.0$ show that the degree of the $c$ axis-orientation increases with increasing $\lambda$. These results indicate that the SAXS meridional peak originated from the $\alpha$-phase lamellae.

WAXS results at $142^{\circ} \mathrm{C}$ show that during the hot roll deformation, $\beta$-phase crystallites are destroyed and then transformed into $c$-axis-oriented $\alpha$-phase crystallites. These results are similar to those observed in the cold roll deformation. The $\beta$-phase reflection of SAXS $Z$-pattern changes from a homogeneous ring at $\lambda=1.0$ to diagonal four arcs at $\lambda=1.3$, and then to elliptical four points at $\lambda=2.0$. This change shows the same tendency in the lamellar deformation mechanism observed in the cold rolling deformation; i.e., in the original specimen, the lamellar normal is distributed homogeneously around the $Z$-axis. At the first stage of the deformation, rotation of lamellae occurs until almost all the lamellae are inclined at about $\pm 45^{\circ}$ to the roll direction. The second stage of deformation proceeds by interlamellar slip and the angle $\phi_{\mathrm{A}}$ consequently increases as observed at $\lambda=2.0$.

In the case of cold rolling, the SAXS meridional reflections appeared when the rolled sample was annealed under tension. In the case of hot rolling at

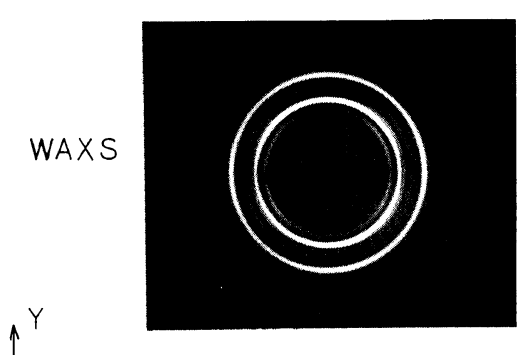

a

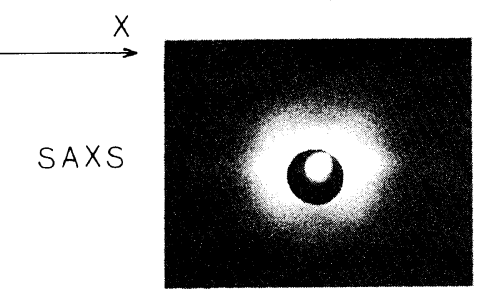

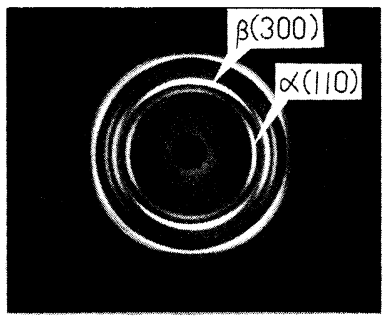

b

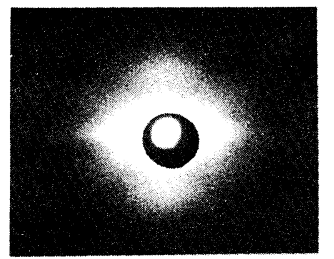

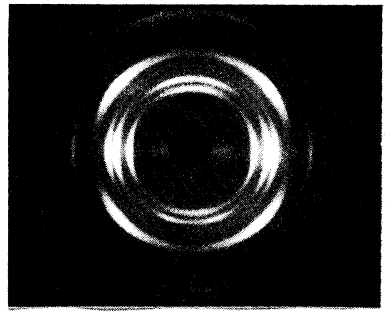

C

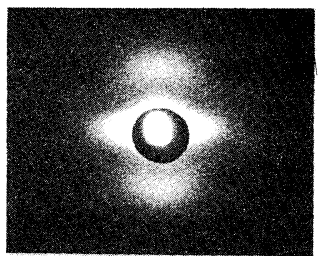

Figure 5. SAXS and WAXS $Z$-patterns from the sample A-rolled at $142^{\circ} \mathrm{C}: \mathrm{a}, \lambda=1.3 ; \mathrm{b}, \lambda=2.0 ; \mathrm{c}, \lambda=3.0$. The roll direction ( $Y$-axis) is vertical. 
$142^{\circ} \mathrm{C}$, these reflections appear during the deformation, showing that hot rolling has both deformation and annealing effects.

\section{B-Roll Deformation}

The results observed at room temperature indicate that, as described in our previous paper, ${ }^{1}$ there are two different mechanisms for the B-roll deformation: In the lamella called F-lamella, whose normal is parallel to the $X$-axis in the original sample (Figure 1), the deformation develops mainly by the chain slip along the chain direction, resulting in a tilting of the $c$-axis in the F-lamella. On the other hand, in the lamella called E-lamella, whose normal is parallel to the $Y$-axis in the original sample, the deformation proceeds by the $(300)<1 \overline{2} 0>$ transversal chain slip without any inclination of the $c$-axis and the lamellar normal. As a consequence of the transversal chain slip or so-called duplex slip, the WAXS $Y$-pattern shows six distinct (300) peaks which have rotated from the original positions by $30^{\circ}$ in the $X Z$ plane as shown in Figure 6a.

In the case of hot rolling, anisotropic behavior in the deformation is also observed with WAXS $X$ - and $Y$-patterns. The WAXS $Y$-pattern from the sample B-rolled at $122^{\circ} \mathrm{C}$ is shown in Figure $6 \mathrm{~b}$, where the reflections from the $\beta$-phase crystals are very weak and the orientation of the $\beta$-phase (300) reflection is inclined at about $\pm 45^{\circ}$ from the $Z$-axis. There are strong $\alpha$-phase $h k 0$ reflections on the equator, indicating that the $\alpha$-phase crystals have $c$-axisorientation with the $c$-axis parallel to the rolling direction $(Z$-axis). The difference between the two $Y$ patterns in Figure 6 shows that at higher temperatures, the $\beta$-phase crystallites are easily destroyed

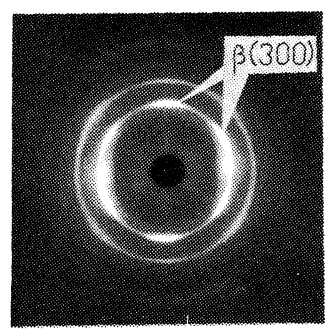

c

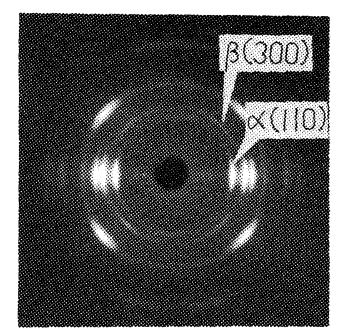

b

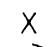

Figure 6. WAXS $Y$-patterns of the sample B-rolled (a) at $20^{\circ} \mathrm{C}, \lambda=3.3$ and (b) at $122^{\circ} \mathrm{C}, \lambda=3.5$. The roll direction $(Z$-axis) is vertical. and transformed into $\alpha$-phase crystallites. In the Elamella, the deformation proceeds by transversal slip. However, the (300) slip plane is inclined at about $45^{\circ}$ from the $Z$-axis and the complete duplex slip, as observed at room temperature, does not appear at $122^{\circ} \mathrm{C}$. On the other hand, the strength and sharpness of the reflections from the $c$-axis-oriented $\alpha$ phase crystallites increase with increasing rolling temperature because of the increasing $\beta \rightarrow \alpha$ transition and the annealing effect at the hot roll temperature. In these B-roll experiments, no $c$-axisoriented $\beta$-phase crystallites could be found in the texture following deformation.

Deformation in the F-lamellae at $68^{\circ} \mathrm{C}$ was observed in the WAXS $X$-pattern, which showed that deformation proceeds mainly by longitudinal chain slip in the same manner as observed at room temperature. However, it was difficult to study the F. lamella deformation at higher temperatures because reflections from $\beta$-phase crystallites became too weak.

In the SAXS $X$ - and $Y$-patterns, meridional reflections appear when the sample is B-rolled above $68^{\circ} \mathrm{C}$, and are considered to originate in the $\alpha$-phase lamellae because their intensity increases with that of WAXS $\alpha$-phase reflections. The long spacing estimated from the SAXS meridional reflections increases with increasing rolling temperature, but does not change with draw ratio. The values of the long spacing for each temperature coincide nearly with those observed for the A-roll deformation.

\section{C-Roll Deformation}

SAXS $Y$-patterns of the sample C-rolled at $122^{\circ} \mathrm{C}$ at stages of $\lambda=1.0, \lambda=1.2, \lambda=1.5$, and $\lambda=2.5$ are shown in Figure 7. In the original sample, a SAXS $Y$ pattern was an equatorial two-point pattern showing that the normal of the F-lamella is parallel to the $X$ axis. At $\lambda=1.2$, four diagonal spots were observed, representing the normal of $F$-lamellae inclined about $45^{\circ}$ with the $X$-axis as a result of interlamellar slip. The diagonal spots at $\lambda=1.5$ and $\lambda=2.5$ show that the angle between the lamellar normal and the $X$-axis increases with the draw ratio. These results are almost the same as those observed at room temperature, indicating that the F-lamellar normal is rotated in the $X Z$ plane as a result of interlamellar slip. The difference between deformations of $F$ - and Elamellae was also observed at the initial stage of the deformation at $122^{\circ} \mathrm{C}$, but it was difficult to measure 


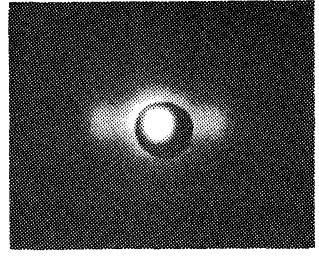

ล

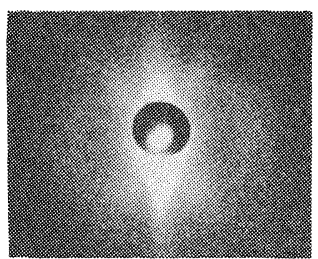

C
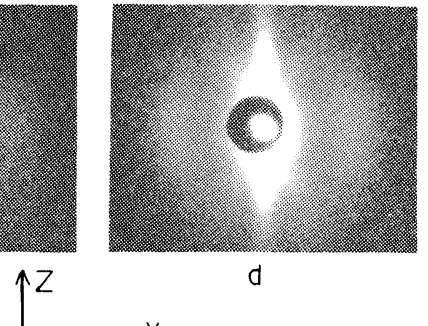

d

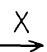

Figure 7. SAXS $Y$-patterns of the sample C-rolled at $122^{\circ} \mathrm{C}$ at various draw-ratios: $\mathrm{a}, \lambda=1.0 ; \mathrm{b}, \lambda=1.2 ; \mathrm{c}$, $\lambda=1.5 ; \mathrm{d}, \lambda=2.5$. The roll direction ( $X$-axis) is horizontal.

the orientation of E-lamellae with SAXS $X$-pattern because the $\beta$-phase reflection was so weak. The difference in deformation was observed also in WAXS patterns. WAXS $Y$-patterns showed that the transversal slip occurs in the E-lamella, but the observation at high temperatures was difficult because of weakness of the $\beta$-phase reflections.

In Figure 7, diffuse peaks are observed on the equator more than $\lambda=1.5$, and seem to arise from $\alpha$ phase lamellae as they appear in accordance with the appearance of the $c$-axis-oriented $\alpha$-phase reflections in the WAXS pattern. The intensity of the equatorial reflections increases with increasing draw ratio. The long spacing measured from the SAXS $\alpha$-phase reflection does not increase with the draw-ratios, but changes according to the roll temperature only, in the same manner as observed for the A- and B-roll deformations. The strong scattering along the meridional direction in Figure 7 seems to originate from voides expanding parallel to the $X Y$ plane formed during the deformation.

\section{DISCUSSION}

The results obtained in hot roll deformation are summarized as follows.

(1) The transition from the $\beta$-phase crystal to the $\alpha$-phase increased with increasing roll temperature and draw ratio.

(2) Anisotropy in deformation was observed in the A-, B-, and C-roll deformations, including rotation of lamellae, interlamellar slip, transversal and longitudinal chain ślip. At higher temperatures, the deformation by the chain slip was not observed to occur, indicating that the $\beta$-phase crystallites are easily destroyed before this slip takes place.

(3) The $c$-axis-oriented $\beta$-phase crystallites were not found in the present hot roll experiments.

(4) The $c$-axis-oriented $\alpha$-phase crystallites appeared during hot roll deformation and formed more developed crystalline lamellae with increasing rolling temperature. The long spacing observed from the SAXS $\alpha$-phase reflection increased with increasing roll temperature.

The remarkable results in hot roll deformation are shown in (1) and (4), while the results of the deformation of the $\beta$-phase crystallites as shown in (2) and (3) are nearly the same as those observed in cold rolling. The results shown in (4) are in good agreement with the deformational behavior observed by drawing and rolling of polyethylene and other polymers. $^{9,10}$

Prior to this experiment, it was expected that the original $\beta$-phase crystal could be found in the final $c$ axis-oriented texture after hot rolling. The results, on the contrary, indicate that the original texture is more easily destroyed at higher temperature. The original $\beta$-phase crystallites are transformed by hot roll deformation as well as by cold roll deformation to the $c$-axis-oriented $\alpha$-phase crystallites. These results give a negative conclusion as to the possibility of incorporating the original texture into the $c$-axisoriented final texture referred to in the Introductory part. However, it is not clear whether these results show the general tendency of polymer deformation or certain special characteristics of the isotactic polypropylene $\beta$-phase crystal.

Samuels, et al., have pointed out that the $\beta \rightarrow \alpha$ phase transition of isotactic polypropylene requires a local unfolding or melting because of the difference in the crystalline structures. ${ }^{6}$ From these considerations, it is supposed that also in the case of hot roll deformation of $\beta$-phase isotactic polypropylene, the original texture transforms, through unfolding or melting and subsequent recrystallization, into $c$-axisoriented $\alpha$-phase crystallites.

Awaya has reported ${ }^{11}$ that the $\beta$-phase reflection 
appears during the drawing of the $\alpha$-phase sample, suggesting that the $\alpha \rightarrow \beta$ transition can take place during deformation. If, as he says, the $\alpha \rightarrow \beta$ transition can take place by a more local rearrangement of molecular segments without partial melting, the above mentioned conclusion requires further examination. Some brief supplementary drawing tests were carried out with $\alpha$-phase samples in our laboratory. However, no appearance of $\beta$-phase crystals was shown. It seems that a more detailed investigation is necessary for this problem.

\section{REFERENCES}

1. T. Asano and Y. Fujiwara, Polymer, 19, 99 (1978).

2. T. Seto, Tokyo Metropolitan University, private communication, 1958.

3. R. Corneliussen and A. Peterlin, Makromol. Chem., 105, 193 (1967).

4. F. J. Baltà-Calleja, A. Peterlin, and B. Crist, J. Polym. Sci., Part A-2, 10, 1749 (1972).

5. O. Yoda and I. Kuriyama, J. Polym. Sci., Polym. Phys. Ed., 15, 773 (1977).

6. R. J. Samuels and R. Y. Yee, J. Polym. Sci., Part A-2, 10, 385 (1972).

7. Y. Fujiwara, Kolloid Z. Z. Polym., 226, 135 (1968).

8. H. D. Keith, F. J. Padden, N. M. Walter, and H. W. Wyckoff, J. Appl. Phys., 30, 1485 (1959).

9. G. Meinel, N. Morosoff, and A. Peterlin, J. Polym. Sci., Part A-2, 8, 1723 (1970).

10. A. Peterlin and F. J. Baltà-Calleja, J. Appl. Phys., 40, 4238 (1969).

11. H. Awaya, Kobunshi Kagaku (Chem. High Polymers), 20, 1 (1963). 